\title{
Biorevitalization of postmenopausal labia majora, the polynucleotide/hyaluronic acid option
}

\author{
Isabella Pia Palmieri ${ }^{1,2}$ and Mauro Raichi ${ }^{3 *}$ \\ ${ }^{1}$ Professor, University of Padua Medical School, Master Diploma in Surgery and Aesthetics, Padua, Italy \\ ${ }^{2}$ Professor, University of Naples Medical School, Master Diploma in Esthetic Medicine, Naples, Italy \\ ${ }^{3}$ Clinical Pharmacology Consultant, University of Milan Medical School, Milan, Italy
}

\begin{abstract}
Background and objectives: The genitourinary syndrome of menopause, with special reference to vulvovaginal atrophy, impacts on postmenopausal women's quality of life. There is a rationale for exploring the effectiveness of vulvovaginal bio-revitalization with techniques similar to those successful in cutaneous bio-revitalization (infiltration and topical application of polynucleotides and hyaluronic acid).

Methods: Prospective cohort study of women with post-menopausal vulvovaginal symptoms for at least 1 year; the interim results for the first 19 enrolled women are presented. Infiltrative bio-revitalization procedure: 3 priming sessions with highly concentrated polynucleotides from salmon trout gonads ( $40 \mathrm{mg} / 2 \mathrm{~mL}$; bi- or three-weekly infiltrations) followed by 2 consolidation sessions with sodium hyaluronate $(40 \mathrm{mg} / 2 \mathrm{~mL})$ bi-weekly. Anesthetic cream applied before all infiltrations. Evaluations: basally and after $21,35,50,70$ and 90 days.

Results: All women experienced rapid symptom improvement after the two first priming infiltrations (symptom scores falling between $42.2 \%$ and $52.6 \%$ vs. baseline), with special reference to itching and soreness, tenderness and tingling (symptom scores, $-52.6 \%$ and $-51.6 \%$, respectively). Dyspareunia and vaginal dryness scores fell by $59.4 \%$ and $59.1 \%$, respectively, at the end of follow-up.

Conclusion: The outcomes of this explorative study might suggest that both the documented bio-reactivating properties of polynucleotides in the skin and the similarly documented synergy between polynucleotides and hyaluronic acid might also be expressed in post-menopausal vulvovaginal tissues, though confirmation by well-designed controlled trials is warranted.
\end{abstract}

\section{Introduction}

The vulvovaginal environment undergoes changes all over life, for instance during the transition from adolescence to sexual maturity and during pregnancy. The menopausal loss of ovarian function associates with the most dramatic of such lifetime changes [1]. Postmenopausal estradiol concentrations are steadily less than $30 \mathrm{pg} / \mathrm{mL}$, compared with premenopausal levels that fluctuate between $10 \mathrm{pg} / \mathrm{mL}$ and $800 \mathrm{pg} / \mathrm{mL}$ depending on when measured during the cycle. Any postmenopausal estradiol derives from estrone, which is peripherally converted in adipose tissue from adrenal androstenedione [1].

Progressive thinning and drying of vaginal mucosa, as well as loss of elasticity and pliability and smoothing of vaginal rugae, are all elements of the phenotypical expression of vulvar and vaginal atrophy (VVA) within the more general framework of the genitourinary syndrome of menopause (GSM) [2]. GSM and its VVA component translate for affected women into a variable set of disturbing vulvar, vaginal, and clitoral symptoms: loss of lubrication, irritation, and itching; dyspareunia and other sexually related problems; dysuria and recurrent urinary tract infections [2].

International surveys show that $39 \%$ to $51 \%$ of postmenopausal women experience vulvar and vaginal symptoms within 4-5 years after menopause, with $55 \%$ to $62 \%$ of symptomatic women reporting moderate-to-severe vaginal symptoms [3]. The situation in Italy seems even more serious, with prevalence of confirmed vulvovaginal symptoms, according to the very recent European Vulvovaginal
Epidemiological Survey (EVES), estimated as high as 75\% in women attending Italian menopausal centers [4]. As all over the world, confirmed GSM associates with more severe symptoms, lower quality of life, and compromised sexual function compared with lack of confirmed vulvovaginal symptoms [4].

Climacteric is only part of the problem since chronologic ageing and, occasionally, rheumatologic disorders contribute to genitourinary tract changes and compromised vulvovaginal esthetics in midlife and older women [2]. Surprisingly, the Women's EMPOWER survey highlights that only $40 \%$ of U.S. women associate vulvo-vaginal symptoms with menopause; awareness seems higher in Europe, according to the Real Women's Views of Treatment Options for Menopausal Vaginal Changes (REVIVE) survey [3].

The Women's EMPOWER survey in the U.S. shows that $41-62 \%$ of women who receive treatment for GSM and vulvovaginal symptoms only use over-the-counter products such as lubricating gels and moisturizers. All such products only provide temporary relief, mainly

${ }^{\star}$ Correspondence to: Mauro Raichi, Clinical Pharmacology Consultant, University of Milan Medical School, Milan, Italy, E-mail: mauro.raichi@gmail.com

Key words: bio-revitalization, genitourinary syndrome of menopause, hyaluronic acid, polynucleotides, vulvovaginal atrophy

Received: October 13, 2019; Accepted: October 24, 2019; Published: October 29,2019 
during sexual activity; $50 \%$ of women never receive any treatment [3]. As a consequence, there is an urgent need for effective and wellaccepted therapeutic options targeting the vulvovaginal symptoms of menopause and ageing without the liabilities of systemic hormone therapy and resistance to local hormone treatment $[5,6]$.

The evidence of accelerated skin ageing after menopause is the rationale for exploring the benefits of infiltrative vulvovaginal mucosal bio-revitalization in postmenopausal women, aiming to increase vulvovaginal hydration and turgidity and to control mucosal dystrophia. The postmenopausal estrogen deficiency in the skin is associated with reduction of collagen, elastin, blood flow, decreased skin thickness, and loss of elasticity and tension; the functional impairment of sebaceous and sweat glands leads to dry rough skin. Depletion of hyaluronic acid skin levels is also paramount [7].

Exploring the esthetic and clinical benefits of vulvovaginal infiltrative bio-revitalization - priming with polynucleotides (PN) to re-activate the deposition of new collagen and elastin fibers and the production of new extra-cellular dermal matrix, followed by hyaluronic acid (HA) as consolidator of initial benefits (more hydration of extracellular matrix, further increase of deposition of new collagen and elastin fibers) - was the rationale for the sequential bio-revitalization protocol in postmenopausal women herein discussed. A noncomparative exploratory study began in 2017; study formulations were similar to skin bio-revitalizing products used in regenerative and esthetic medicine. The results of this exploratory study, ended in May 2019, are herein illustrated; a more rigidly designed controlled study is beginning, based on validated assessments of GSM symptoms and sexual wellbeing of women.

\section{Materials and Methods}

\section{Prospective study cohort}

Nineteen women in physiological or surgical menopause, aged between 50 and 65 years, with lack of menses for at least one year and experiencing postmenopausal vaginal dryness, mucosal atrophy confirmed by the investigator (thinning or loss of vaginal rugation, mucosal pallor and petechiae, tenderness of the vaginal introitus, etc.), as well as other GSM symptoms including dyspareunia. Genitourinary infections, pelvic organ prolapse beyond the hymenal ring, vulvodynia or chronic vulvar pain related to vulvar dermatitis or dystrophy, viral lesions including high risk for human Papillomavirus infections, positive Papanicolau test, hormone replacement therapy, and any local treatment aimed at vulvovaginal symptom control were the exclusion criteria. Menopause was natural at 45-53 years in 17 women (late menopause in one woman at 56 years) and surgical because of total hysterectomies in the previous four years in 2 women. As regards comorbidities, 1 woman suffered from Sjögren syndrome; 14 women had normal pregnancies (parity, 1-3); 1 woman reported a spontaneous miscarriage. After screening, all women of the prospective cohort provided written informed consent to anonymous collection of their data before the first vulvovaginal bio-revitalization session. All study materials, including informed consent forms, study protocol and case report forms, were peer-reviewed for ethical problems; the study followed the principles of the Declaration of Helsinki and was carried out at the medical and surgical facilities of the post-graduate school of the Italian Society of Esthetic Medicine and Surgery (CPMA), Bologna, Italy.

\section{Vulvovaginal bio-revitalization protocol, priming sessions}

Labial infiltration in the superficial lamina propria of atrophic labia majora of $2 \mathrm{~mL}$ of a highly purified mixture of polynucleotides (40 $\mathrm{mg}$ ) of controlled mean molecular weights from salmon trout gonads ("primer"; PN concentration of the prefilled syringe: $40 \mathrm{mg} / 2 \mathrm{~mL}$; infiltration depth: about $3 \mathrm{~mm}$ below the vulvar skin surface); 3 sessions every either 2 or 3 weeks. The needle of the prefilled syringe was $30 \mathrm{G}$ and was inserted with a 30-45 degrees inclination. PN infiltrations were performed with the linear retrograde technique in labia majora; with the micro-wheal technique in labia minora and around the vaginal meatus and clitoris $(0.1 \mathrm{~mL}$ per wheal; distance between wheals, $0.5-1 \mathrm{~cm})$.

\section{Vulvovaginal bio-revitalization protocol, consolidation sessions}

Labial infiltration in the formerly "primed" areas of $2 \mathrm{~mL}$ of a solution of highly purified natural-origin hyaluronic acid as sodium hyaluronate ("consolidator": molecular weight, 1000-1500 kDa; concentration $40 \mathrm{mg} / 2 \mathrm{~mL}$ ); 2 sessions every 2 weeks starting 15 days after the last PN session with the same infiltration technique used in the previous phase. A galenic anesthetic cream (30\% lidocaine gel prepared by a local pharmacy for all women) was applied to the areas to be infiltrated 30 minutes before the procedure; the amount was the smallest needed to numb the target vulvar skin (usually about $1 \mathrm{~cm}$ ). Figure 1 illustrates the infiltration points on labia majora. Infiltrations were performed linearly and retrogradely, beginning with no more than two infiltrations below the posterior labial commissure overlying the perineal body and one or two infiltrations at the anterior labial commissure below the mons pubis. What is left in the syringe is infiltrated in several small hives on labia minora at the side of the vestibule and up to the prepuce: patients often comment this second part of the procedure is modestly painful, yet bearable. All products are commercially available as Class III medical devices (CE0373 - Newest ${ }^{\oplus} 10 \mathrm{mg} / \mathrm{ml} \mathrm{PN}$ and $10 \mathrm{mg} / \mathrm{ml}$ HA fixed combination with mannitol), PN alone formulation (CE0373 Plinest $^{\oplus} 20 \mathrm{mg} / \mathrm{ml} \mathrm{PN}$ ), HA alone formulation (CE0373 - Ialest ${ }^{\circledR} 20 \mathrm{mg} / \mathrm{ml}$, mean HA molecular weight about $1,250 \mathrm{kDa}$ ) as isotonic viscoelastic gels in prefilled, single-use sterile and apyrogenic syringes; $30 \mathrm{G} / 13 \mathrm{~mm}$ needles.

Suggested supportive treatment: Topical at-home application of polynucleotides, formulated as either vaginal pessaries or cream.

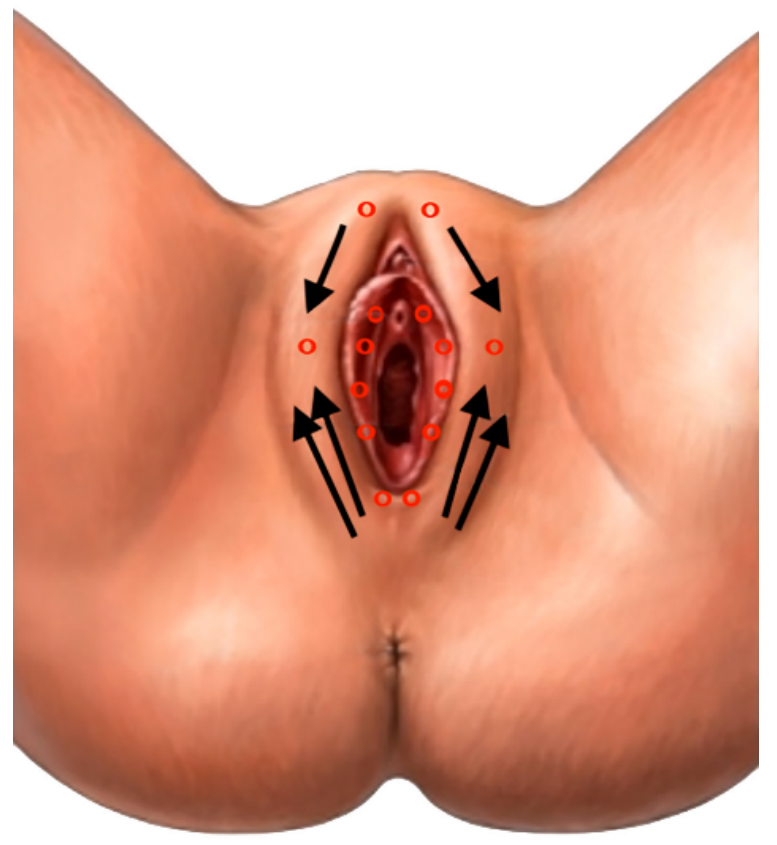

Figure 1. Infiltration points on labia majora with woman on the gynaecological chair 


\section{Clinical assessments, timing, and parameters}

Demographics and scoring of leading vulvovaginal symptoms (vaginal dryness; irritation; itching; soreness, tenderness and tingling; dyspareunia) were pre-planned at basal evaluation (T0) and after 21, 35, 50, 70 and 90 days (T21, T35, T50, T70 and T90, respectively). Ten-cm visual analogue scales (VAS) were to be used by women for symptom scoring $(0=$ no symptom, $10=$ unbearable symptom). Vulvoperineal adiposity, tissue hydration, hair density and distribution, overall morphology of labia majora and minora, leukorrhea, vulvar skin texture, fissures and other lesions were also to be assessed by vulvar inspection by the investigator at T0 and T90. Secondary GSM symptoms (vaginal discharge, dysuria, frequency, urge incontinence, recurrent urinary tract infections) and vulvovaginal esthetics were not formally assessed and analyzed, and were only informally discussed with women during the study (secondary GSM symptoms) and at the final visit (vulvovaginal esthetics). The investigator carefully looked for complications of the procedure at all study visits.

\section{Statistics}

Descriptive statistics were generated for demographics and physical examination findings. The nonparametric Wilcoxon Signed Rank Test and the Kolmogorov-Smirnov test for repeated measurements (continuous variables) on single populations were applied to VAS mean scores. Two-sided 95\% confidence levels were used for all statistical tests with $\mathrm{p}<0.05$ as cut-off for significance. Statistical software: IBM Statistical Product and Service Solutions (SPSS), version 24.

\section{Results}

Less than half of the cohort women reported dysuria, urge incontinence or other secondary GSM symptoms at basal evaluation, in all cases clinically mild. All secondary symptoms and overall esthetics qualitatively improved during follow-up with less irritation and dryness of vulvar skin as well as less paleness, dryness and atrophy of vulvovaginal mucosae; however, no formal statistical analysis of clinical scores was performed.

All women of the prospective cohort completed the study; compliance to infiltrations, assessed with structured interviews at all evaluation times, was always excellent and all women completed the planned priming (PN) and consolidation (HA) sessions. Mean age of women at T0 was 56.9 years, mean weight $65.0 \mathrm{~kg}$; vulvovaginal symptoms in a 54-year-old teacher were confirmed as related to her known Sjögren syndrome.

All women of the prospective cohort experienced highly significant and rapid improvements in GSM symptoms after the two first priming PN infiltrations; symptoms scores fell between $42.2 \%$ and $52.6 \%$ compared with baseline (Figure 2 and Table 1). Symptom improvement vs. baseline after the first and the second PN infiltrative session (T21, T35), and between the baseline and the last (T90) consolidating infiltration, are shown in Table 2. No complication of the biorevitalization protocol was observed during the 90-day study period.

All women qualitatively reported at least some improvement of secondary GSM symptoms (vaginal discharge, dysuria, frequency, urge incontinence, recurrent urinary tract infections): only in four women at T21 visit, in most women (15 out of 19) at T50 visit, and in all women at the final T70 interview. All women qualitatively reported at least a good level of satisfaction for vulvovaginal esthetic outcomes at follow-up.

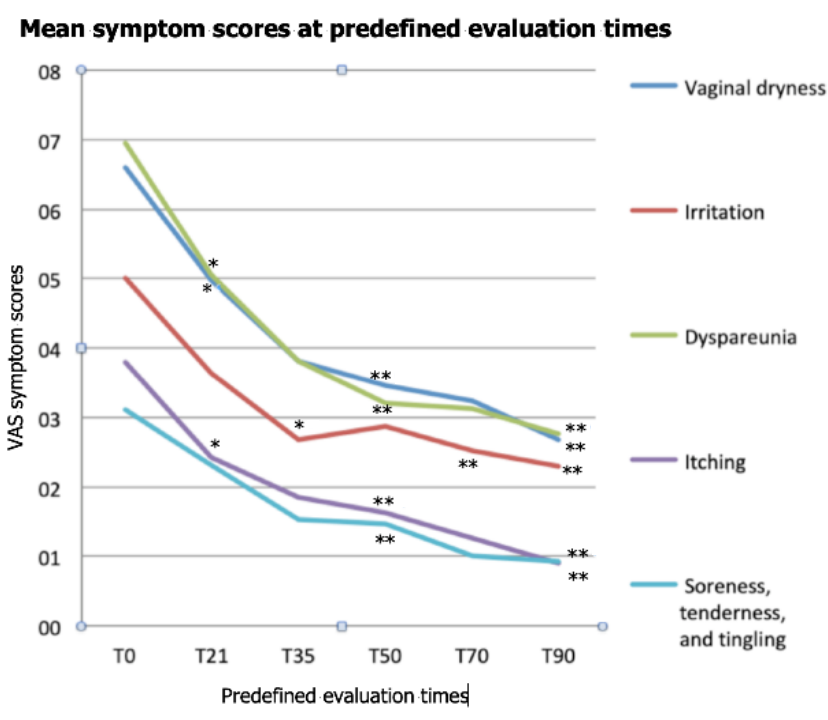

Figure 2. Evolution of VVA mean symptom scores over the 90-day follow-up period. "p $<0.05$ vs. baseline; "* $p<0.01$ vs. baseline

Table 1. Mean (top line) and standard error of the mean (SEM, lower line) at assessment times during the study

\begin{tabular}{|c|c|c|c|c|c|c|}
\hline & T0 & $\mathbf{T 2 1}$ & T35 & T50 & T70 & T90 \\
\hline \multirow[t]{2}{*}{ Vaginal dryness } & 6.6 & $5.0^{*}$ & 3.8 & $3.5^{* *}$ & $3.2^{* *}$ & $2.7^{* * *}$ \\
\hline & 0.24 & 0.30 & 0.23 & 0.24 & 0.3 & 0.16 \\
\hline \multirow[t]{2}{*}{ Irritation } & 5.0 & 3.6 & $2.7^{*}$ & $2.9^{*}$ & $2.5^{* *}$ & $2.3^{* *}$ \\
\hline & 0.40 & 0.36 & 0.30 & 0.23 & 0.22 & 0.19 \\
\hline \multirow[t]{2}{*}{ Dyspareunia } & 6.9 & $5.1^{*}$ & $3.8^{*}$ & $3.2^{* *}$ & $3.1^{* * *}$ & $2.8^{* *}$ \\
\hline & 0.34 & 0.34 & 0.26 & 0.24 & 0.24 & 0.16 \\
\hline \multirow[t]{2}{*}{ Itching } & 3.8 & $2.4^{*}$ & 1.8 & $1.6^{* *}$ & $1.3^{* * *}$ & $0.9^{* * *}$ \\
\hline & 0.38 & 0.35 & 0.34 & 0.17 & 0.13 & 0.17 \\
\hline \multirow[t]{2}{*}{$\begin{array}{l}\text { Soreness, tenderness } \\
\text { and tingling }\end{array}$} & 3.1 & 2.3 & 1.5 & $1.5^{* *}$ & $1.0^{* * *}$ & $0.9^{* *}$ \\
\hline & 0.48 & 0.41 & 0.33 & 0.18 & 0.17 & 0.19 \\
\hline
\end{tabular}

Table 2. Improvements of mean GSM symptom scores after the first and second PN priming sessions (T21, T35) and after the second HA consolidation session, and the end of the follow-up period (T90) vs. baseline

\begin{tabular}{|l|c|c|c|}
\hline \multicolumn{4}{|l|}{ Percent variations at selected assessment times vs. baseline } \\
\hline Vaginal dryness & T21 & T35 & T90 \\
\hline Irritation & $-24.2 \%$ & $-42.4 \%$ & $-59.1 \%$ \\
\hline Dyspareunia & $-28.0 \%$ & $-46.0 \%$ & $-54.0 \%$ \\
\hline Itching & $-26.0 \%$ & $-44.9 \%$ & $-59.4 \%$ \\
\hline Soreness, tenderness and tingling & $-36.8 \%$ & $-52.6 \%$ & $-76.3 \%$ \\
\hline
\end{tabular}

\section{Conclusion}

GSM symptoms impact on the woman's self-perception and selfconfidence; they also severely challenge the quality of life of affected women [8]. Loss of labia majora adiposity, hair and pigmentation, blundering of interlabial sulci, and reduced density of sebaceous and sweat glands are all phenotypical expressions of postmenopausal vulvar involution; preputial retraction frequently leads to clitoral exposure and chronic irritation, and the cervix may become flush with the vaginal wall [9]. Microscopically, the peri- and postmenopausal fall of estrogens and reduced blood flow progress hand in hand with matrix glycosaminoglycan depletion, fragmentation and fusion of elastin fibers, and collagen hyalinization [9]. Epithelial thinning and dystrophia leads 
to loss of the normal acidic environment, rise of vaginal $\mathrm{pH}$ beyond the 4.6-threshold, and an overall dysfunctional vaginal ecosystem [1,2]. Alpha and beta estrogen receptors are diffused throughout the urethral and peri-urethral connectives, bladder trigone and pelvic muscles, and this explains why urogenital symptoms - urgency, frequency, nocturia, urge incontinence, microscopic hematuria, recurrent urinary tract infections - are commonly associated with the main vulvovaginal symptoms within the larger framework of GSM. Urogenital atrophic symptoms may be seen even in $15 \%$ of premenopausal women [10].

Chronologic vulvovaginal ageing and systemic diseases - in the treatment cohort, problems were associated with Sjögren disease in one woman - are also important contributors, justifying the current explosion of interest for esthetic (cosmetic) gynecology. In Europe, reductive labiaplasty procedures doubled in the United Kingdom in the decade around the turn of the century [11], whilst 3,300 Italian women underwent plastic surgery in 2014 (1.3\% of all esthetic surgery procedures, $+13 \%$ vs. 2013 ), according to the Italian Association for Aesthetic Plastic Surgery (AICPE) [12].

Polynucleotides, a mixture of purines, pyrimidines, deoxyribonucleotides, and deoxyribonucleosides, have long been known to have a stimulating effect on trophism of dermal cells and tissues [13]. Twenty years ago PN first demonstrated to enhance proliferation of human fibroblasts [13]. PN were also shown to facilitate the repair of UVB-damaged dermal fibroblasts and to accelerate repair of skin ulcers $[13,14]$. PN might also promote the proliferation of human pre-adipocytes, an effect that could help to counteract the loss of labial adiposity [13]. Extensively used in esthetic medicine for rejuvenation of skin, PN fillers replenish the contracted or depressed spaces and may help to improve the regeneration of several autologous skin fractions, e.g., glycosaminoglycans, proteins, and fibrils [15]. Beyond regenerative dermatology, PN have already shown benefits in gynecological surgery and orthopedics [16,17]. All these activities are related to PN acting to enrich tissue of nucleotides and nucleosides, their elementary constituents, and indirectly helping to streamline the metabolism of connectives [18,19].

Due to the shared ectodermal origin, it would not be surprising if PN showed the same trophic effect seen in the skin also in vulvar tissues. This was indeed the rationale that led to identify PN as the primer in the sequential bio-revitalization protocol herein discussed.

Highly concentrated HA was identified as the agent of choice to consolidate the benefits of PN priming. HA restores the hydration crucial for fibroblast trophism [20]: HA has already demonstrated to potentiate the skin bio-revitalization effects of PN [13]. In agreement with the HA hydrating effects that help to restore turgidity, elasticity, and tonicity in the skin, several studies have investigated the benefits of HA dermal fillers to counteract the loss of elasticity and volume of female external genitalia in women with mild to moderate labia majora hypotrophy [21,22].

Due to the exploratory nature of the study, the investigators avoided quantitative scoring with validated scoring instruments, as well as any formal analysis of GSM secondary symptoms and overall vulvar esthetics. This first study on the new bio-revitalization strategy deliberately concentrated on the personal experience of women and their self-assessed GSM symptoms. The handy $10-\mathrm{cm}$ VAS tool was chosen because of high reproducibility of outcomes and ease of use with minimum training; moreover, the VAS tool has already been extensively used in GSM studies including in breast cancer survivors [23].
The authors are aware that using non-validated VAS scales instead of specific and validated tools may weaken the scientific interest of the study. Their choice was primarily due to the very preliminary, probing nature of the investigation. The study was originally to be carried out only in a few informed women, in full compliance with the Helsinki Declaration and ethical requirements in human studies, but only as a very preliminary exploration of the "PN priming" concept. The study then progressed up to the prospective cohort herein described. Such unplanned expansion of the study aimed to further confirm the earliest unexpected positive results. Another reason that induced the authors to use non-validated VAS scoring system is the paucity of patient-based, culturally sensitive, validated instrument for assessing GSM symptoms and their impact on women's lives. One such instrument is the recently validated Vulvovaginal Atrophy Questionnaire (VVAQ) [24], but unfortunately there is still no Italian-language validated translation of the questionnaire. The lack of an independent evaluator of treatment outcomes is another weakness of the study.

All the five main vulvovaginal symptoms underwent rapid improvements during the priming period with $\mathrm{PN}$; this was especially true for itching, soreness, tenderness and tingling, with relevant scores falling at T35 by $52.6 \%$ and $51.6 \%$, respectively. Possibly PN rapidly restored the epithelial and dermal dystrophic defects and microscopic erosions that are known to be associated with vulvovaginal atrophy $[1,2]$. Almost all women reported (qualitatively) at least some improvement in severity of secondary GSM symptoms and esthetic satisfaction between the T21 and T50 visits, but no formal quantitative assessments of either secondary GSM symptoms or vulvovaginal esthetics were performed, and not even a tentative conclusion can be proposed about these issues.

The symptomatic response of vaginal dryness was somewhat slower during the priming period (score, $-42.4 \%$ ), but consistently improved during the HA consolidation period (scores, $-51.5 \%$ at T70 and $-59.1 \%$ at T90). The same was observed for vulvovaginal irritation (scores, $-46 \%$ at $\mathrm{T} 35,-50 \%$ at $\mathrm{T} 70$ and $-54 \%$ at $\mathrm{T} 90)$. It may be discussed if such delayed improvements were mainly related to slow synergy between PN and HA rather than to the later, strong HA hydrating effect. Possibly both hypotheses are true and the answer has to wait for further, more in-depth studies. The same evolution was observed for irritation and dyspareunia, unsurprisingly since vaginal dryness, irritation, and painful sexual intercourses have intuitively much in common.

Another important point to discuss is why vaginal dryness also improved. Infiltrations were performed in vulvar labia majora and minora (Figure 1): the basis for improving of vaginal dryness may seem feeble. Possibly the concomitant topical application of the PN as the topical cream or pessaries acted as a confounding factor that prevented to enucleate the pure effects of vulvar infiltrations. Our preliminary results suggest that a topical $\mathrm{PN}$ vaginal treatment might effectively complement the effects of vulvar infiltrations, but further better designed study are warranted to clarify this point.

Summarizing, PN priming seems both effective and rapid in alleviating the symptoms related to postmenopausal GSM, atrophy, and ageing; much of the overall improvement was already observed after the first weeks of the follow-up period (T35 symptom scores fell between $42.2 \%$ and $52.6 \%$ compared to baseline). Informally, several women reported a welcome improvement of their sexual life and couple relationship. The treatment cohort, though small, was not homogeneous in terms of age of menopause and parity of treated women, meaning that the illustrated considerations might likely be valid for the whole spectrum of menopausal women. Of course, any conclusion should take into consideration the lack of a control group. 
This bias can be acceptable in an exploratory study, but any conclusion must be considered only a suggestion until more solidly designed studies are available.

An interesting point is the strong, early improvement of itching and of soreness, tenderness and tingling (T35 scores, $-52.6 \%$ and $-51.6 \%$, respectively), with further steady progression until late in the HA consolidation period (T90 scores, $-76.3 \%$ and $-70.1 \%$, respectively). These symptoms possibly have a neuropathic component due to a functional damage of the afferent lamina propria innervation. Might PN have a neurotrophic, or at least neuroprotective action in the vulvovaginal mucosa? Further studies are warranted to answer this interesting question.

This study is the first one to suggest that GSM symptoms and compromised esthetics might benefit from a bio-revitalization treatment with $\mathrm{PN}$ and $\mathrm{HA}$ as, respectively, primer and consolidator. Even in an exploratory study, lack of a control group is a liability, yet improvements in subjective symptom control and vulvar esthetics were quite real. Long-term, randomized studies are warranted to confirm these preliminary encouraging results. These future studies will also have to include validated evaluations of the women's distress related to sexual dysfunction and loss of sexual gratification.

\section{Conflicts of interest}

The authors have been R\&D consultants to Mastelli S.r.l., Sanremo, Italy (PN patent holder and producer). They certify that at present they have no conflict of interest with any financial or commercial organization in relation to the content of this manuscript.

\section{Acknowledgements}

The authors wish to thank the PN manufacturer for providing the products needed for the study.

\section{References}

1. Mac Bride MB, Rhodes DJ, Shuster LT (2010) Vulvovaginal atrophy. Mayo Clin Proc 85: 87-94.

2. Mitchell CM, Waetjen LE (2018) Genitourinary changes with ageing. Obstet Gynecol Clin North Am 45: 737-750.

3. Krychman M, Graham S, Bernick B, Mirkin S, Kingsberg SA (2017) The women's EMPOWER survey: women's knowledge and awareness of treatment options for vulvar and vaginal atrophy remains inadequate. $J$ Sex Med 14 :425-433.

4. Nappi RE, Seracchioli R, Salvatore S, Cagnacci A, Di Paolantonio T, et al. (2019) Impact of vulvovaginal atrophy of menopause: prevalence and symptoms in Italian women according to the EVES study. Gynecol Endocrinol 35: 453-459.

5. Marjoribanks J, Farquhar C, Roberts H, Lethaby A (2017) Long-term hormone therapy for perimenopausal and postmenopausal women. Cochrane Database Syst Rev 1: CD004143.

6. Kingsberg SA, Krychman ML (2013) Resistance and barriers to local estrogen therapy in women with atrophic vaginitis. $J$ Sex Med 10: 1567-1574.
7. Galęba A, Bajurna B, Shankar S (2014) Premature skin ageing - a process that can be slowed down by appropriate prevention. Hygeia Public Health 49: 708-711.

8. Portman D, Gass M; Vulvovaginal Atrophy Terminology Consensus Conference Panel (2014) Genitourinary syndrome of menopause: new terminology for vulvovaginal atrophy from the International Society for the Study of Women's Sexual Health and The North American Menopause Society. Menopause 21: 1063-1088.

9. Sturdee DW, Panay N; International Menopause Society Writing Group (2010) Recommendations for the management of postmenopausal vaginal atrophy. Climacteric 13: 509-522.

10. Palacios S (2009) Manageing urogenital atrophy. Maturitas 63: 315-318.

11. Liao LM, Creighton SM (2007) Requests for cosmetic genitoplasty: how should healthcare providers respond? Br Med J 334: 1090-1092.

12. Italian Association for Plastic Aesthetic Surgery (AICPE), Italian Association for Aesthetic and Functional Gynaecology (AIGEF). Joint Session, 2nd National AICPE Congress, 14-16 March 2014, Florence (Italy).

13. Guizzardi S, Uggeri J, Belletti G, Cattarini G (2013) Hyaluronate increases polynucleotides effect on human cultured fibroblasts. J Cosm Dermatol Sci Applic 3: 124-128.

14. De Caridi G, Massara M, Acri I, Zavettieri S, Grande R, et al. (2016) Trophic effects of polynucleotides and hyaluronic acid in the healing of venous ulcers of the lower limbs: a clinical study. Int Wound $J$ 13: 754-758.

15. Cavallini M, Papagni M (2007) Long chain polynucleotides gel and skin biorevitalization. J Plastic Dermatol 3: 27-32.

16. Giani I, Elbetti C, Novelli E, Zanieri DG (2013) Polynucleotides and terpinenol: an effective aid in preventing mesh exposure in pelvic floor surgery. Pelvi-Perineologia 32: 77-79.

17. Dallari D, Sabbioni G, Del Piccolo N, Carubbi C, Veronesi F, et al. (2018) Efficacy of intra-articular polynucleotides associated with hyaluronic acid versus hyaluronic acid alone in the treatment of knee osteoarthritis: a randomized, double-blind, controlled clinical trial. Clin J Sport Med 1.

18. Gennero L, Denysenko T, Calisti GF, Vercelli A, Vercelli CM, et al. (2013) Protective effects of polydeoxyribonucleotides on cartilage degradation in experimental cultures. Cell Biochem Funct 31: 214-227.

19. Squadrito F, Bitto A, Irrera N, Pizzino G, Pallio G, et al. (2017) Pharmacological activity and clinical use of PDRN. Front Pharmacol 8: 224.

20. Landau M, Fagien S (2015) Science of hyaluronic acid beyond filling: fibroblasts and their response to the extracellular matrix. Plast Reconstr Surg 136: 1888-1958.

21. Fasola E, Gazzola R (2016) Labia majora augmentation with hyaluronic acid filler: technique and results. Aesthet Surg J 36: 1155-1163.

22. Zerbinati N, Haddad RG, Bader A, Rauso R, d'Este E, et al. (2017) A new hyaluronic acid polymer in the augmentation and restoration of labia majora. $J$ Biol Regul Homeost Agents 31: 153-161.

23. Lee YK, Chung HH, Kim JV, Park N, Song Y, et al. (2011) Vaginal pH-balanced gel for the control of atrophic vaginitis among breast cancer survivors: a randomized controlled trial. Obstet Gynecol 117: 922-927.

24. Shifren J, Portman D, Krychman M (2017) The Vulvovaginal Atrophy Questionnaire (VVAQ): a novel patient-reported Outcome (PRO) for assessing symptoms of vulvovaginal atrophy in menopausal women.

Copyright: (C2019 Palmieri IP. This is an open-access article distributed under the terms of the Creative Commons Attribution License, which permits unrestricted use, distribution, and reproduction in any medium, provided the original author and source are credited. 